xiv

\title{
A Formula in the Theory of Correlation
}

\author{
By A. E. Hope.
}

The formula in question gives the correlation between two variables for a number $n_{1}+n_{2}$ of individuals when the ordinary parameters, including correlations, are available for the separate groups of $n_{1}$ and $n_{2}$ individuals; and it is extended to more than two groups. It is possible, even probable, that the formula is known, but the writer has not found any direct references to it; in any case its practical value is such that there can be no harm in drawing attention to it.

In the first place there is the well known formula connecting the standard deviation $\sigma$ with the corresponding root-mean-square deviation $s$ about a "provisional mean" differing by $h$ units from the true mean. It is

$$
s^{2}=\sigma^{2}+h^{2}
$$

Next, suppose two groups of $n_{1}$ and $n_{2}$ individuals measured respectively with regard to some character $x$, the means being $m_{1}$ and $m_{2}$. Let $m$ be the mean of the whole group $n=n_{1}+n_{2}$. Then

$$
m=\frac{n_{1} m_{1}+n_{2} m_{2}}{n_{1}+n_{2}}
$$

Let $\sigma_{1}, \sigma_{2}$ be the standard deviations of the two groups, $\sigma$ that of the whole group. Then

and similarly for $n_{2} \sigma_{2}^{2}$.

$$
\begin{aligned}
n_{1} \sigma_{1}^{2} & =\Sigma\left(x-m_{1}\right)^{2} \\
& =\Sigma\left(x-m+m-m_{1}\right)^{2},
\end{aligned}
$$

The "total" squared deviation of the group $n_{1}+n_{2}$ is

$$
\left(n_{1}+n_{2}\right) \sigma^{2}
$$

derived partly from $\sigma_{1}^{2}$ and partly from $\sigma_{2}^{2}$, these, however, being modified by being taken from $m$ as origin. Hence we have

where

$$
\left(n_{1}+n_{2}\right) \sigma^{2}=n_{1} s_{1}^{2}+n_{2} s_{2}^{2}
$$

$$
\begin{aligned}
& s_{1}^{2}=\sigma_{1}^{2}+\left(m-m_{1}\right)^{2}, \\
& s_{2}^{2}=\sigma_{2}^{2}+\left(m-m_{2}\right)^{2}, \text { by }(1) .
\end{aligned}
$$

From (2) we find

$$
m-m_{1}=n_{2}\left(m_{2}-m_{1}\right) /\left(n_{1}+n_{2}\right), \quad m-m_{2}=n_{1}\left(m_{1}-m_{2}\right) /\left(n_{1}+n_{2}\right) .
$$


Now writing $m_{1}-m_{2}=h$, we obtain from (3)

$$
\begin{aligned}
\left(n_{1}+n_{2}\right) \sigma^{2} & =n_{1} \sigma_{1}^{2}+n_{2} \sigma_{2}^{2}+\frac{n_{1} \frac{n_{2}^{2} h^{2}}{\left(n_{1}+n_{2}\right)^{2}}+\frac{n_{1}^{2} n_{2} h^{2}}{\left(n_{1}+n_{2}\right)^{2}}}{}=n_{1} \sigma_{1}^{2}+n_{2} \sigma_{2}^{2}+n_{1} n_{2} h^{2} /\left(n_{1}+n_{2}\right),
\end{aligned}
$$

which gives $\sigma^{2}$ in terms of known quantities.

Again, let $m_{1 x}$ and $\sigma_{1 x}$ denote the mean and s.d. of group $n_{1}$ in regard to $x$, with $m_{2 x}$ and so on for the group $n_{2}$, and similar notation with regard to $y$. Let $r_{1}$ and $r_{2}$ be the correlation coefficients of $x$ and $y$ in the groups, $r$ that in the whole group; $m_{x}, m_{y}$ the "total" means, $\sigma_{x}, \sigma_{y}$ the corresponding s.d.'s; $h_{x}$ the difference of means of groups in $x ; h_{y}$ similarly; $\Sigma_{1}, \Sigma_{2}$ summations over the groups, $\Sigma$ the “total" summation. Then

Now

$$
r=\frac{\Sigma\left(x-m_{x}\right)\left(y-m_{y}\right)}{\left(n_{1}+n_{2}\right) \sigma_{x} \sigma_{y}}
$$

$$
\begin{aligned}
\Sigma_{1}\left(x-m_{x}\right)\left(y-m_{y}\right) & =\Sigma_{1}\left(x-m_{1 x}+m_{1 x}-m_{x}\right)\left(y-m_{1 y}+m_{1 y}-m y\right) \\
& =\Sigma_{1}\left(x-m_{1 x}\right)\left(y-m_{1 y}\right)+n_{1} n_{2}^{2} h_{x} h_{y} /\left(n_{1}+n_{2}\right)^{2},
\end{aligned}
$$

after a little working. Adding this to the corresponding result involving $\Sigma_{2}$, we derive

$$
\begin{gathered}
\Sigma\left(x-m_{x}\right)\left(y-m_{y}\right)=\Sigma_{1}\left(x-m_{1 . x}\right)\left(y-m_{1 y}\right)+\Sigma_{2}\left(x-m_{2, x}\right)\left(y-m_{2 y}\right) \\
+\frac{n_{1} n_{2}}{n_{1}+n_{2}} h_{x} h_{y}
\end{gathered}
$$

or, by definition,

$$
\left(n_{1}+n_{2}\right) r \sigma_{x} \sigma_{y}=n_{1} r_{1} \sigma_{1 x} \sigma_{1 y}+n_{2} r_{2} \sigma_{2 x} \sigma_{2 y}+\frac{n_{1} n_{2}}{n_{1}+n_{2}} h_{x} h_{y} .
$$

The values of $\sigma_{x}, \sigma_{y}$ being already given in (4), we have finally

$r=\frac{n_{1} r_{1} \sigma_{1 x} \sigma_{1 y}+n_{2} r_{2} \sigma_{2 x} \sigma_{2 y}+n_{1} n_{2} h_{x} h_{y} /\left(n_{1}+n_{2}\right)}{\left[n_{1} \sigma_{1 x}^{2}+n_{2} \sigma_{2 x}^{2}+n_{1} n_{2} h_{x}^{2} /\left(n_{1}+n_{2}\right)\right]^{\frac{1}{2}}\left[n_{1} \sigma_{1 y}^{2}+n_{2} \sigma_{2 y}^{2}+n_{1} n_{2} h_{y /}^{2} /\left(n_{1}+n_{2}\right)\right]^{\frac{1}{2}}}$,

which is the formula required.

The structure of the formula appears most clearly in the extension to any number of pooled groups, which is not difficult to establish. It is then

$$
r=\frac{\sum n_{i} r_{i} \sigma_{i x} \sigma_{i y}+\left(\Sigma n_{i} n_{j} h_{i j x} h_{i j y}\right) / \Sigma n_{i}}{\left[\Sigma n_{i} \sigma_{i x}^{2}+\left(\Sigma n_{i} n_{j} h_{i j x}^{2}\right) / \Sigma n_{i}\right]^{\frac{1}{2}} \cdot\left[\Sigma n_{i} \sigma_{i y}^{2}+\left(\Sigma n_{i} n_{j} h_{i j y}^{2}\right) / \Sigma n_{i}\right]^{\frac{1}{2}}} .
$$


xvi

For practical workings with the formula (8) a useful variant is obtained by employing not standard deviations but "variances" $V$ defined by

$$
n \sigma^{2}=c^{2} V
$$

where $c$ is the "class interval." We then have

$$
\begin{aligned}
n_{1} \sigma_{1 x}^{2} & =c_{1 x}^{2} V_{1 x}, \text { etc., } \\
n_{1} r_{1} \sigma_{1 x} \sigma_{1 y} & =r_{1} c_{1 x} c_{1 y} \sqrt{ }\left(V_{1 x} V_{1 y}\right)
\end{aligned}
$$

The reader may be left to make the substitution, which takes a specially useful form when, as is normally the case, the class intervals for both groups in $x$, as well as in $y$, are the same.

\section{The Probability Distribution of a Bridge Hand}

By J. B. Marshall.

The probability distribution of a bridge hand affords a good example of drawings without replacement from a limited stock.

Let $n$ drawings be made from such a stock. Let $p_{r s}$ and $q_{r s}$ be the probabilities of success and failure after there have been $r$ drawings with $s$ successes, and let the probabilities in successive drawings be connected by the relation

$$
p_{r s} q_{r+1, s+1}=q_{r \delta} p_{r+1, s}
$$

[This relation is easily seen to hold in the case of a bridge hand. For if $b$ is the number of cards left in the pack after $r$ drawings, and if $a$ is the number which will give a successful result, then

whence

$$
p_{r s}=a / b, \quad p_{r+1, s}=a /(b-1), \quad p_{r+1, s+1}=(a-1) /(b-1),
$$

$$
\begin{aligned}
p_{r s} q_{r+1, s+1} & =\frac{a}{b} \times \frac{(b-1)-(a-1)}{b-1} \\
& =\frac{b-a}{b} \times \frac{a}{b-1} \\
& \left.=q_{r \delta} p_{r+1, s .}\right]
\end{aligned}
$$

Let us, in the usual manner, construct a generating function (G.F.) by introducing a variable $t$, the powers of which will enumerate 